\title{
Brain and bone marrow metastases from rectal cancer
}

\author{
Konstantina Thomopoulou*, Stavroula Manolakoua*, Ippokratis Messaritakis ${ }^{\mathrm{b}}$, Maria Tzardic, \\ Eleni Lagoudakic, Anastasios Koutsopoulosc, Andreas Koulourisa, George Kanellis d, \\ Konstantinos Kalbakis ${ }^{a}$, Dimitris Mavroudis ${ }^{\text {, }}$ Ioannis Souglakos ${ }^{a}$
}

University Hospital of Heraklion, Crete; University of Crete; Evangelismos Hospital, Athens, Greece

\begin{abstract}
Despite the development of new treatment options based on the molecular characterization of colorectal cancer, $20 \%$ of patients present de novo metastatic disease, whereas $30-40 \%$ of patients who receive curative treatment relapse during follow up. Herein, we report 2 cases with rectal cancer that developed uncommon sites of metastasis; the first patient had an isolated breast metastasis, while the second patient developed bone marrow infiltration with synchronous brain metastases. In order to evaluate the uncommon metastatic pattern of rectal cancer, we detected and enumerated circulating tumor cells (CTCs) using both immunofluorescence and real-time reverse transcriptase polymerase chain reaction in these patients' peripheral blood. The procedure revealed the presence of CTCs, positive for CEACAM5 but negative for epithelial phenotype (EpCAM-), that might explain the patients' metastatic potential and survival.
\end{abstract}

Keywords Rectal cancer, rare metastases, circulating tumor cells

Ann Gastroenterol 2019; 32 (6): 1-3

\section{Introduction}

Despite developments in the management of colorectal cancer (CRC), eventually $20 \%$ of patients present de novo metastatic disease, whereas $30-40 \%$ of patients relapse after curative colectomy. The median overall survival of patients with metastatic CRC (mCRC) has increased significantly over the past decade and reaches almost 3 years [1]. The most common metastatic sites of relapse are the lung and the liver [2].

Herein, we report 2 rectal cancer cases with extremely uncommon sites of metastasis: in one patient isolated breast

\footnotetext{
${ }^{\mathrm{a}}$ Medical Oncology Department, University Hospital of Heraklion, Crete (Konstantina Thomopoulou, Stavroula Manolakou, Andreas Koulouris, Konstantinos Kalbakis, Dimitris Mavroudis, Ioannis Souglakos); bLaboratory of Translational Oncology, Faculty of Medicine, University of Crete (Ippokratis Messaritakis); 'Department of Pathology, University Hospital of Heraklion, Crete (Maria Tzardi, Eleni Lagoudaki, Anastasios Koutsopoulos); 'Hematopathology Department, Evangelismos Hospital, Athens (George Kanellis), Greece

${ }^{\star}$ Equal contribution

Conflict of Interest: None
}

Correspondence to: Ioannis Souglakos, PAGNI Hospital, 71110 Voutes, Heraklion, Crete, Greece, e-mail: johnsougl@gmail.com

Received 25 July 2019; accepted 6 September 2019; published online 8 October 2019

DOI: https://doi.org/10.20524/aog.2019.0423 metastasis and in the other bone marrow (BM) infiltration with synchronous brain metastasis.

\section{Case 1}

A 58-year-old female, with a medical history of hyperthyroidism and dilated cardiomyopathy and a family history of a first-degree relative with CRC at an early age, underwent a wide local excision of a rectal mass due to a pT1 low-grade adenocarcinoma, initially found on screening endoscopy. The patient had 3-monthly clinical examination and carcinoembryonic antigen (CEA) testing, and 6-monthly computed tomography (CT) scans of thorax and abdomen. At 30 months of follow up, a rising CEA level was detected and the CT showed 3 pulmonary nodules, histologically confirmed as mCRC. Detection of circulating tumor cells (CTCs) using immunofluorescence and real-time reverse transcriptase polymerase chain reaction revealed the presence of CEACAM5+/EPCAM-CTCs. The tumor harbored a KRAS G13D mutation and had a proficient mismatch repair system. The patient received oxaliplatin-based chemotherapy and after 4 cycles she presented a partial response (reduction in lung nodules $>50 \%$ ) and no other metastatic sites were detected by positron emission tomography or CT. The patient underwent a wide excision of all lung metastases. Five years later, she noticed a lump in her right breast (Fig. 1). Core biopsy revealed a breast metastasis of primary rectal cancer and she underwent right mastectomy with axillary node dissection. Histology was consistent with rectal metastasis with spread to 
1 of 13 lymph nodes. She is on follow up with no evidence of occurrence.

\section{Case 2}

An 80-year-old female with a history of arterial hypertension presented with a T3bN2M0 rectal carcinoma with a positive circumferential margin on magnetic resonance imaging (MRI). The patient received neoadjuvant oxaliplatinbased chemotherapy in the context of a phase II clinical trial and after 6 cycles of chemotherapy she underwent low anterior resection. Histology showed a T3N1c undifferentiated rectal adenocarcinoma, with poor response to neoadjuvant chemotherapy. CTC detection revealed the presence of CEACAM5+/EPCAM-CTCs. The patient received 5 cycles of 5-fluorouracil/leucovorin postoperatively because of neurotoxicity. Six months later, the she presented with acute blurred vision and left hemiparesis. Brain MRI revealed one parieto-occipital lesion with malignant features (Fig. 2). Surgical excision of the mass was performed, and the histology revealed mCRC with invasion of excised meninx (Fig. 3A-D). After the

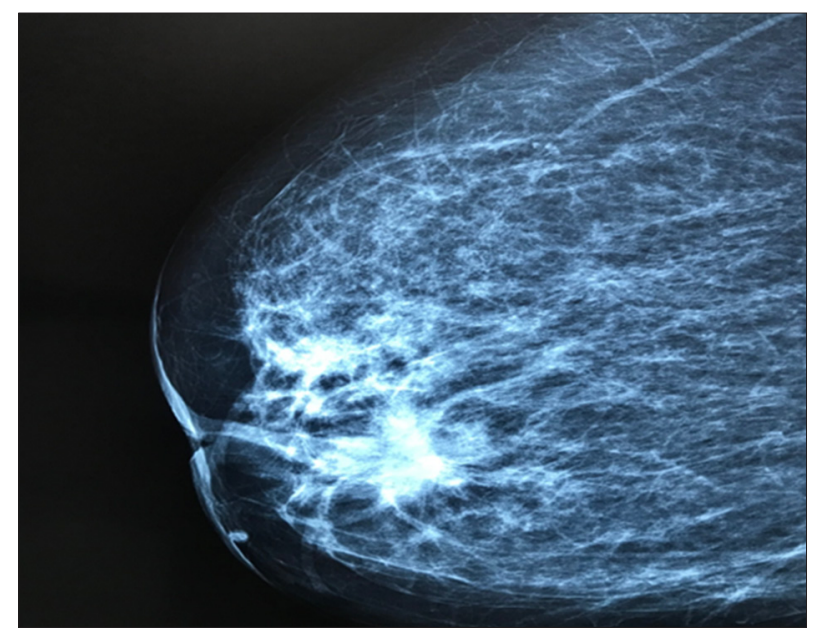

Figure 1 Mammography showing a breast mass measuring $11 \times 10 \mathrm{~cm}$

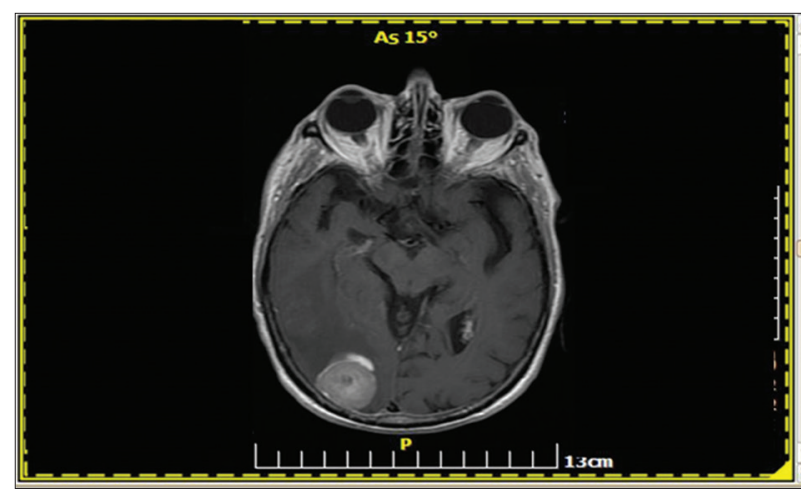

Figure 2 Magnetic resonance imaging of the brain (T2-FLAIR sequence) revealed one parieto-occipital lesion with malignant features operation, she developed persistent grade 3 thrombocytopenia and grade 2 anemia and she underwent BM aspiration and biopsy. Histology showed massive BM infiltration from mCRC (Fig. 4A-D). The patient died 3 months later as a result of sepsis from resistant urinary tract infection.

\section{Discussion}

We present 2 rectal cancer cases with rare metastatic sites. The first case, originally diagnosed with a stage I, low-grade adenocarcinoma, developed a single breast metastasis many years later, while the second patient, originally diagnosed as stage III, high-grade rectal cancer, relapsed rapidly with $\mathrm{BM}$ infiltration and a single brain metastatic lesion. In the literature, rectal cancer with breast and BM metastases signifies a highly aggressive nature of malignancy associated with a poor prognosis, given that the 1 -year survival is $<20 \%$ [3]. The different $\mathrm{T}$ status and histopathologic characteristics and the secondary BM tumor infiltration may explain the difference in their outcomes. A Swiss retrospective study demonstrated that bone marrow micrometastasis in stage I-III colon cancer was a negative prognostic factor for relapse and survival [2]. Moreover, in a retrospective study with 17,811 rectal cancer patients, the outcome depended on the mucinous or signet ring characteristics and $\mathrm{T}$ and $\mathrm{N}$ status at diagnosis [4]. The presence of CEACAM5+/EPCAM-CTCs in both patients may also explain the increase metastatic potential and their survival. In fact, as reported previously by our group, the detection of CTCs was a factor of poor prognosis, correlated with a poor outcome and metastatic spread [5]. It is also well recognized that CTCs undergo epithelial-to-mesenchymal transition during their hematogenous dissemination, as a prerequisite for invasion and metastasis, and thus fail to express epithelial surface antigens, such as EpCAM40 [6]. The fact that both patients presented CTCs with a loss of epithelial phenotype highlights their clinical relevance in representing the biological behavior of the tumor more appropriately.

Central nervous system metastases have been assessed as uncommon $(<5 \%)$, while metastatic BM infiltration and breast metastases (incidence $0.5-2 \%$ ) have been recorded only as case reports. For this reason, the differential diagnosis of primary neurological and hematological malignancies or a primary breast cancer may be challenging and the diagnostic workup should be also include the probability of metastasis for rectal cancer [7].

Notably, there is no evidence concerning the treatment management of rectal patients with rare metastatic localizations and it has not yet been proved that metastasectomies confer a survival benefit. Indeed, it has been reported that both BM and breast metastases from rectal cancer are a sign of disseminated disease and chemotherapy alone may have a crucial role [8]. Moreover, several case reports of brain metastasis from colorectal cancer with a curative intent approach showed that a combination of local therapy-i.e., metastasectomy and/or whole brain radiotherapy or stereotactic radiosurgery-with systemic chemotherapy may have a role [9]. As far as breast 


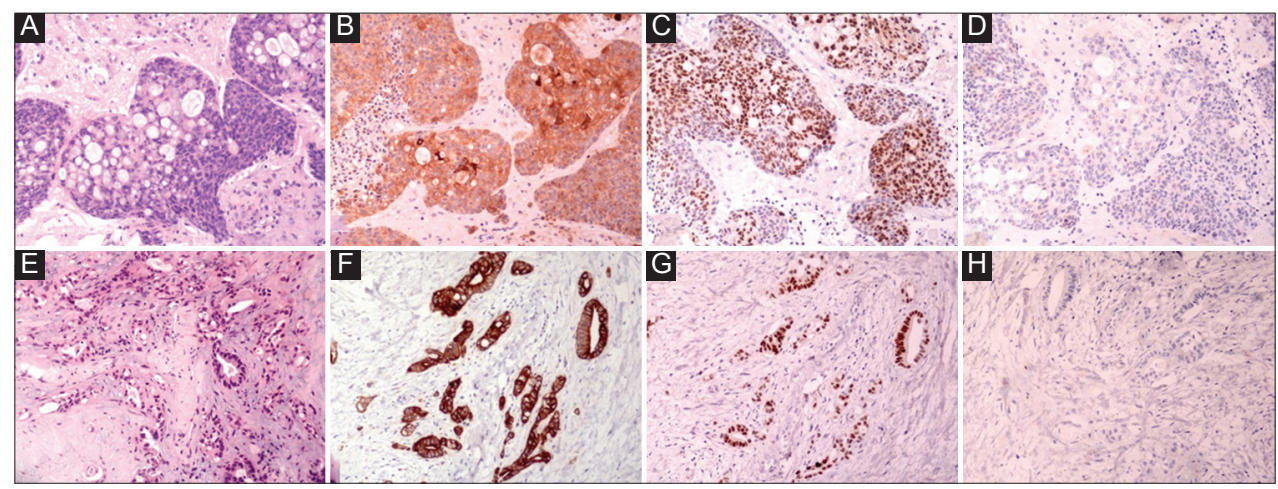

Figure 3 (A-D) Tissue sections from brain metastasis stained for (A) H\&E, (B) CK20, (C) CDX-2 and (D) CK7. E-H: Representative sections (H\&E, E) from surgical specimen of mastectomy showed histopathologic characteristics of a moderately differentiated adenocarcinoma with the following immunophenotype: (F) CK20+, (G) CDX-2+ and (H) CK7. The immunophenotype of both cases was consistent with metastatic colorectal carcinoma (magnification $\times 200$ )

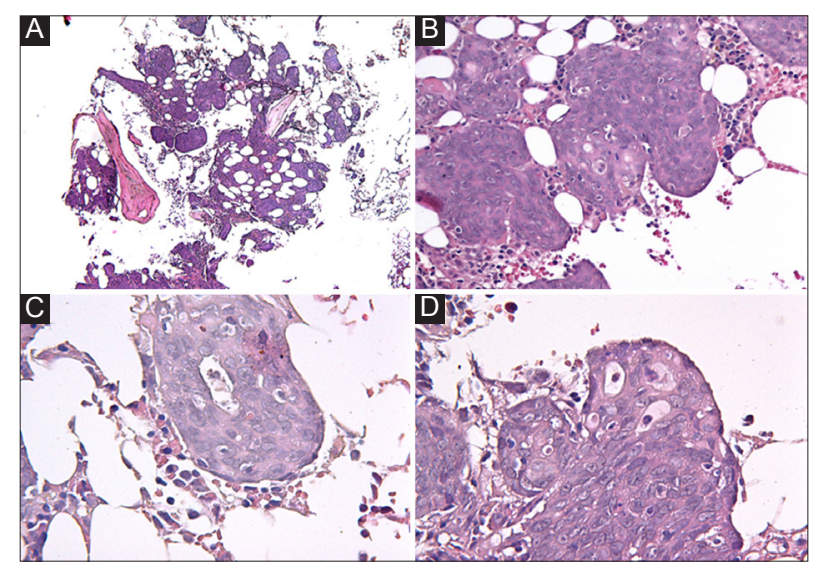

Figure 4 (A-D) Extended infiltration of bone marrow by an adenocarcinoma with almost complete effacement of hematopoietic lineages. Cell morphology consists of large cells with abundant cytoplasm, which form solid nests (B) and in some areas present with atypical glandular differentiation (C \& D)

metastasis treatment is concerned, there are only limited reports describing controversial strategies. Mammary metastasectomy is described as a possible treatment option, although Barthelmes et al argued that surgery should not be performed in view of the short overall survival and the complication of seeding [6]. Furthermore, it remains controversial whether sentinel lymph node biopsy is essential [10].

In conclusion, we have described 2 rectal cancer patients with unusual metastatic sites. Early recognition and diagnosis of rare metastases from rectal cancer is necessary to evaluate the disease's aggressiveness and biological behavior, as well as to form an optimal individualizing treatment plan. However, questions still remain about the surgical and oncological management of these rare conditions and the therapeutic plan should be driven by multidisciplinary cancer teams.

\section{References}

1. Stintzing S, Miller-Phillips L, Fischer von Weikersthal L, et al. Per protocol analysis and final OS update of the FIRE-3 (AIO KRK-0306) study comparing FOLFIRI plus cetuximab vs. FOLFIRI plus bevacizumab. J Clin Oncol 2018;36(Suppl):3508-3508.

2. Disibio G, French SW. Metastatic patterns of cancers: results from a large autopsy study. Arch Pathol Lab Med 2008;132:931-939.

3. Viehl CT, Weixler B, Guller U, et al. Presence of bone marrow micrometastases in stage I-III colon cancer patients is associated with worse disease-free and overall survival. Cancer Med 2017;6:918-927.

4. Riihimäki M, Hemminki A, Sundquist J, Hemminki K. Patterns of metastasis in colon and rectal cancer. Sci Rep 2016;6:29765.

5. Messaritakis I, Sfakianaki M, Papadaki C, et al. Prognostic significance of CEACAM5mRNA-positive circulating tumor cells in patients with metastatic colorectal cancer. Cancer Chemother Pharmacol 2018;82:767-775.

6. Polyak K, Weinberg RA. Transitions between epithelial and mesenchymal states: acquisition of malignant and stem cell traits. Nat Rev Cancer 2009;9:265-273.

7. Williams SA, Ehlers RA $2^{\text {nd }}$, Hunt KK, et al. Metastases to the breast from nonbreast solid neoplasms: presentation and determinants of survival. Cancer 2007;110:731-737.

8. Barthelmes L, Simpson JS, Douglas-Jones AG, Sweetland HM. Metastasis of primary colon cancer to the breast - leave well alone. Breast care Breast Care (Basel) 2010;5:23-25.

9. Kim DY, Ryu CG, Jung EJ, Paik JH, Hwang D. Brain metastasis from colorectal cancer: a single center experience. Ann Surg Treat Res 2018;94:13-18.

10. Perin T, Canzonieri V, Memeo L, Massarut S. Breast metastasis of primary colon cancer with micrometastasis in the axillary sentinel node: a metastasis that metastasized? Diagn Pathol 2011;6:45. 